\title{
Métodos de análise da cinza insolúvel em ácido utilizada como indicador na determinação da energia metabolizável do milho para aves
}

\author{
Analytical methods of acid-insoluble ash used as marker to determining the \\ metabolizable energy of corn for poultry
}

\author{
CARVALHO, Genilson Bezerra de ${ }^{1}$; DOURADO, Leilane Rocha Barros ${ }^{2} *$; \\ LOPES, João Batista ${ }^{2}$; FERREIRA, Antônio Hosmylton Carvalho ${ }^{1}$; RIBEIRO, Mabel \\ Nery'; SILVA, Sandra Regina Gomes da ${ }^{1}$; MERVAL, Ramon Rêgo ${ }^{1}$; BIAGIOTTI, \\ Daniel $^{1}$; SILVA, Francisco Eduardo Soares ${ }^{3}$
}

\author{
${ }^{1}$ Universidade Federal do Piauí, Centro de Ciências Agrárias, Programa de Pós-Graduação em Zootecnia, \\ Teresina, Piauí, Brasil. \\ ${ }^{2}$ Universidade Federal do Piauí, Centro de Ciências Agrárias, Departamento de Zootecnia, Teresina, \\ Piauí, Brasil. \\ ${ }^{3}$ Medico Veterinário, Autonômo, Teresina, Piauí, Brasil. \\ *Endereço para correspondência: leilane@ufpi.edu.br
}

\section{RESUMO}

Objetivou-se avaliar métodos de análise da cinza insolúvel em ácido como indicador na determinação da energia metabolizável do milho para aves em relação ao procedimento padrão de coleta total de excretas. Foi formulada uma dieta referência a base de milho e farelo de soja e uma dieta teste com inclusão de $40 \%$ de milho à dieta referência. Foi adicionado $1 \%$ de celite ${ }^{\mathbf{T M}}$ às dietas, como fonte de cinza insolúvel em ácido. Foram utilizados 60 pintos de corte, da linhagem $\operatorname{Cobb}^{500}$, dos 12 aos 21 dias de idade, distribuídos em um delineamento experimental inteiramente casualizado com duas dietas e seis repetições de cinco aves cada. A energia metabolizável da dieta referência e dieta teste foram determinadas utilizando quatros métodos de análise de cinza insolúvel em ácido (indicador) e também utilizando as fórmulas preconizadas pelo procedimento de coleta total de excretas. Não houve diferença significativa na energia metabolizável do milho determinada com a cinza insolúvel em ácido como indicador quando comparada ao procedimento de coleta total de excretas. Entretanto, observou-se que os métodos de análise da cinza insolúvel em ácido proporcionaram grande variabilidade nos resultados de energia metabolizável do milho, que apresentaram desvio padrão oscilando entre 88 a $229 \mathrm{kcal} / \mathrm{kg}$. A cinza insolúvel em ácido pode ser determinada por vários métodos e utilizada como indicador para estimar a energia metabolizável do milho.

Palavras-chave: digestibilidade, metabolismo animal, metodologia, nutrição.

\section{SUMMARY}

The objective was to evaluate methods of analysis of acid-insoluble ash (AIA) as an indicator for the measurement of apparent metabolisable energy (AME) on corn for poultry compared with the standard technique of total collection of excreta. Was formulated reference diet (RD) based on corn and soybean meal and a test diet (DT) with inclusion of $40 \%$ corn to the RD. It was added $1 \%$ of Celite ${ }^{\mathrm{TM}}$ to the diets as a source of acid-insoluble ash. We used a total of 60 chicks, $C_{o b b}{ }^{500}$, from 12 to 21 days of age, distributed in a completely randomized design, with two treatments and six repetitions with five birds each. The AME of the RD and DT were determined using four methods of analysis of AIA (indicator) and using the formulas recommended by the method of total excreta collection. There wasn't significant difference in AME of corn determined using the AIA as an indicator when compared to the method of total excreta 
collection. However, it was observed that the methods of analysis gave the AIA great variability in results AME of corn that had standard deviations ranging from 88$229 \mathrm{kcal} / \mathrm{kg}$. The acid insoluble ash (AIA) can be determined for most techniques and used to estimate the AME of corn.

Keywords: animal metabolism, digestibility, methodology, nutrition.

\section{INTRODUÇÃO}

A importância do conhecimento do valor nutritivo dos alimentos, assim como da utilização dos nutrientes na obtenção do máximo potencial produtivo e reprodutivo do animal é inquestionável. $\mathrm{O}$ valor nutritivo do alimento é importante no balanceamento das rações e está diretamente relacionado com sua composição química e energética (D'AGOSTINI et al., 2004).

O milho é o principal ingrediente energético utilizado nas rações de aves, seu valor energético médio é conhecido, entretanto algumas variações podem ocorrer dependendo da variedade, do tipo de solo, clima e temperatura de secagem. A energia é um dos fatores nutricionais mais importantes por interferir diretamente no desempenho, e no custo da alimentação (FREITAS et al., 2006).

Foram desenvolvidos vários métodos diretos e indiretos, por meio de ensaio de metabolismo gerando diferentes terminologias para expressar os valores de energia sendo a energia metabolizável aparente (EMA), a mais utilizada na formulação de ração de aves (FREITAS et al., 2006).

A determinação da digestibilidade pelo procedimento tradicional de coleta total de excretas requer controle rigoroso da ingestão e da excreção, o que torna o trabalho mais oneroso. Assim têm-se proposto métodos indiretos de estimativa de excreção fecal e digestibilidade, entre eles, o uso dos indicadores, o qual apresenta vantagens sobre a coleta total de excretas, pela simplicidade e conveniência de utilização (TORRES et al., 2009).

Os indicadores (óxido crômico, Lipe, CIA, etc...) têm sido testados para estimativa da produção de matéria seca fecal e digestibilidades. A cinza insolúvel em ácido (CIA) é um composto mineral indigestível, principalmente sílica, tratada com ácido clorídrico, sendo considerada um indicador interno, entretanto, como o conteúdo de CIA é baixo em grãos, e eventualmente pode ser adicionadas fontes externas de CIA (Celite ${ }^{\mathrm{TM}}$, areia e sílica) às dietas para melhorar a precisão das estimativas, vindo a ser também considerado como um indicador externo (SALES \& JANSSENS, 2003).

O método analítico é um fator que tem grande destaque quando objetiva-se estimativas mais acuradas da energia metabolizável, assim, torna-se evidente a necessidade de avaliar metodologias analíticas com o uso de cinza insolúvel em ácido como indicador externo na estimativa de digestibilidade de nutrientes para aves.

Objetivou-se avaliar métodos de análise da cinza insolúvel em ácido (CIA) como indicador, na determinação da energia metabolizável do milho para aves.

\section{MATERIAL E MÉTODOS}

O experimento foi conduzido no Setor de Avicultura do Departamento de Zootecnia do Centro de Ciências Agrárias da Universidade Federal do Piauí, em Teresina - Piauí.

Para a determinação da EMA do milho foram utilizados 60 pintos de corte 
machos, da linhagem $\operatorname{Cobb}^{500}$, dos 12 aos 21 dias de idade. As aves foram distribuídas aleatoriamente em doze parcelas de cinco aves cada, sendo seis para fornecimento de dieta referência e seis para o fornecimento de dieta teste.

Foi formulada uma dieta referência (DR) a base de milho e farelo de soja para atender as exigências nutricionais das aves (ROSTAGNO et al., 2005), e uma dieta teste com inclusão de $40 \%$ de milho à DR. Foi adicionado $1 \%$ de Celite $^{\mathrm{TM}}$ às dietas, como fonte de cinza insolúvel em ácido, conforme demonstrado na Tabela 1. As aves receberam água e ração à vontade durante todo o período experimental.

Tabela 1. Composição química e percentual das dietas experimentais ${ }^{1}$

\begin{tabular}{|c|c|c|}
\hline \multirow{2}{*}{ Ingredientes $(\%)$} & \multicolumn{2}{|c|}{ Dietas $(\%)$} \\
\hline & Referência (DR) & Dieta Teste (DT) \\
\hline Dieta referência & - & 59,400 \\
\hline Milho & 57,640 & 39,600 \\
\hline Farelo de Soja & 35,150 & - \\
\hline Óleo de Soja & 2,150 & - \\
\hline Sal & 0,427 & - \\
\hline DL-Metionina 99 & 0,275 & - \\
\hline L-Lisine $\mathrm{HCl}$ & 0,230 & - \\
\hline Calcário Calcítico & 0,890 & - \\
\hline Fosfato Bicálcico & 1,833 & - \\
\hline Suplemento Vitamínico $^{2}$ & 0,200 & - \\
\hline Suplemento Mineral ${ }^{3}$ & 0,200 & - \\
\hline Celite $^{\mathrm{TM} 4}$ & $1,000^{*}$ & 1,000 \\
\hline Total & 100,000 & 100,000 \\
\hline \multicolumn{3}{|l|}{ Composição nutricional } \\
\hline EMA (kcal/kg) & 2974 & 3137 \\
\hline PB (\%) & 20,92 & 15,72 \\
\hline Cálcio (\%) & 0,898 & 0,551 \\
\hline Fósforo disponível (\%) & 0,450 & 0,300 \\
\hline Sódio (\%) & 0,218 & 0,142 \\
\hline Lisina digestível (\%) & 1,191 & 0,795 \\
\hline Met + Cis digestível (\%) & 0,847 & 0,636 \\
\hline \multicolumn{3}{|c|}{ 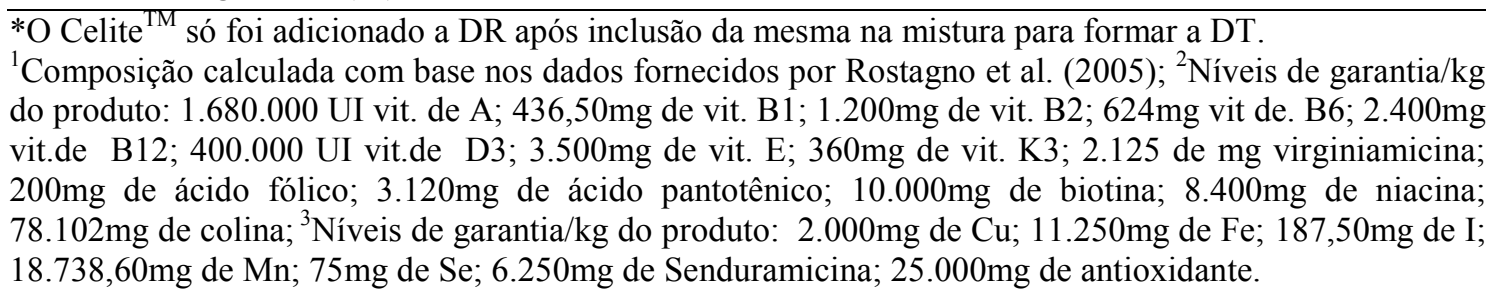 } \\
\hline
\end{tabular}

Do primeiro ao $11^{\circ}$ dia de idade, as aves foram alojadas em galpão de alvenaria, boxe de $3 \times 1 \mathrm{~m}$, com piso de cimento, pé direito de 2,80m de altura, cobertura com telhas de cerâmica, provido de lanternim, muretas laterais de $0,60 \mathrm{~m}$ de 
altura, sendo o restante fechado com telas de arame liso, cortinas plásticas com abertura de baixo para cima. A cama utilizada foi de casca de arroz, com aproximadamente oito centímetros de altura. $\mathrm{O}$ ambiente do interior do galpão foi controlado por cortinas laterais, nebulizadores e ventiladores.

As aves foram pesadas e distribuídas em 12 gaiolas de metabolismo com dimensões de $60 \times 80 \times 55 \mathrm{~cm}$, providas de comedouros e bebedouros. No inicio do período de adaptação às dietas experimentais, foram instaladas bandejas sob as gaiolas, previamente revestidas com plástico para evitar perdas de excretas. $\mathrm{O}$ peso médio inicial das aves no inicio da fase de adaptação foi de $177 \mathrm{~g}$ para aves que receberam dieta referência e $177,83 \mathrm{~g}$ para aves que receberam dieta teste.

O ensaio teve duração de 10 dias, sendo cinco dias para a adaptação às dietas experimentais e cinco para a coleta das excretas. Após o período de adaptação, foi iniciada a coleta das excretas, utilizando óxido férrico (1\%) na ração, como marcador do início e do final da coleta. Durante todo o período experimental, o intervalo entre coletas foi de 12 horas. As excretas foram acondicionadas em sacos plásticos, devidamente identificadas por repetição e armazenadas a $-20^{\circ} \mathrm{C}$ após cada coleta. No final do período experimental, foram determinadas, por repetição, a quantidade de ração consumida e a quantidade total de excretas produzidas. Para a análise do indicador (CIA) uma amostra de aproximadamente $10 \%$ das excretas foi coletada aleatoriamente das bandejas para representar a coleta parcial. O peso destas foi contabilizado como parte da produção total de excretas pelo procedimento de coleta total.

Para a determinação da amostra seca ao ar das excretas, estas foram descongeladas, homogeneizadas por repetição, pesadas e colocadas em estufa de ventilação forçada, à temperatura de $55^{\circ} \mathrm{C}$, por 72 horas. Após a pré-secagem, as amostras foram processadas em moinho tipo faca, com peneira de três $\mathrm{mm}$ e encaminhadas ao laboratório, junto com amostras das rações experimentais, para as análises bromatológicas.

Todas as amostras foram analisadas para obtenção dos teores de matéria seca, energia bruta, e cinza insolúvel em ácido pelos diferentes métodos. A matéria seca foi determinada segundo Silva \& Queiroz (2002). Para a determinação da energia bruta, as amostras foram peletizadas, e submetidas à combustão em bomba calorimétrica (1281, PARR, Instruments, EUA).

$\mathrm{O}$ delineamento experimental foi $\mathrm{O}$ inteiramente casualizado com cinco tratamentos (energia metabolizável aparente (EMA) estimada pelo procedimento de coleta total (Tratamento 1) e pelo uso de cinza insolúvel em ácido como indicador (Tratamento 2 a 5) através do método de coleta parcial determinada com quatro técnicas analíticas) e seis repetições. As análises químicas foram realizadas em duplicata e a média foi utilizada para compor a repetição de acordo com a amostra analisada.

A cinza insolúvel em ácido foi determinada por quatro métodos, sendo que os métodos I e II foram adaptados da metodologia descrita por Santos et al. (2008) e os métodos III e IV foram adaptados da metodologia descrita por Van Keulen \& Young (1977).

No método I foi utilizado aproximadamente $0,75 \mathrm{~g}$ de amostra préseca (ração ou excreta), pesadas em tubos cônico de borosilicato com capacidade para $15 \mathrm{~mL}$. Após pesagem os tubos permaneceram em estufa de 
secagem a $80^{\circ} \mathrm{C}$ durante 12 horas e então foram incineradas em forno mufla com temperatura de $450^{\circ} \mathrm{C}$ por 10 horas. Após esfriamento foi adicionado a cada tubo com amostra incinerada, $5 \mathrm{~mL}$ de ácido clorídrico $4 \mathrm{~N}$ e estes foram levadas ao bloco digestor a $125^{\circ} \mathrm{C}$, permanecendo nesta temperatura por 30 minutos. As amostras permaneceram em descanso até $o$ completo esfriamento e então, foram centrifugadas por 10 minutos a 1500rpm. O ácido foi descartado e em cada tubo com amostra foi adicionado aproximadamente $3 \mathrm{~mL}$ de água destiladadeionizada, agitadas em aparelho vortex e novamente centrifugadas pelo mesmo tempo e rotação. $\mathrm{O}$ procedimento de lavagem com a água foi repetido por três vezes consecutivas e após todas as amostras passarem por este procedimento, foram colocadas em estufa de secagem a $80^{\circ} \mathrm{C}$ por 12 horas. Após esse período, novamente, as amostras retornaram ao forno mufla a $450^{\circ} \mathrm{C}$ por 10 horas, sendo procedida, após o esfriamento das amostras em dessecador, a pesagem para a determinação da porcentagem de cinza ácida insolúvel.

O método II foi uma modificação do método I, pela redução do número de lavagens com água deionizada para uma lavagem com $5 \mathrm{~mL}$ de água deionizada, e também redução do numero de horas (de 12 para 4h) na estufa após a digestão com $\mathrm{HCl}$ e lavagem.

No método III foram utilizadas $10 \mathrm{~g}$ de amostra de dieta e de excreta, pesadas em cadinhos de $100 \mathrm{~mL}$ e incineradas em forno mufla por cinco horas com aumento progressivo da temperatura até atingir $600^{\circ} \mathrm{C}$. À cinza resultante foi adicionado $5 \mathrm{~mL}$ de água e $10 \mathrm{~mL}$ de $\mathrm{HCl}$ concentrado $(11$ a $12 \mathrm{~N})$ mantidos em banho maria até completa evaporação (esse procedimento foi repetido duas vezes). Em seguida mais
$5 \mathrm{~mL}$ de $\mathrm{HCl}$ concentrado foi adicionado, e após $15 \mathrm{~min}$ foram filtrados (Whatman n. 42). As cinza digeridas foram lavadas com água destilada aquecida e posteriormente transferidas para cadinhos e levadas à mufla por aproximadamente 10 a $12 \mathrm{~h}$ à $650^{\circ} \mathrm{C}$. Após o esfriamento das amostras em dessecador, foi procedida a pesagem para a determinação da porcentagem de cinza insolúvel em ácido.

No método IV foram utilizadas $10 \mathrm{~g}$ de amostra (dieta ou excreta), pesadas em Erlenmeyer de $500 \mathrm{~mL}$ e adicionados $100 \mathrm{~mL}$ de $\mathrm{HCl} 4 \mathrm{~N}$. Essa mistura foi mantida em placa aquecida por $30 \mathrm{mim}$. Posteriormente o conteúdo aquecido foi filtrado (Whatman n. 42) e lavado com água destilada aquecida e posteriormente transferidas para cadinhos e levadas à mufla por aproximadamente 10 a $12 \mathrm{~h}$ à $650^{\circ} \mathrm{C}$. Após o esfriamento das amostras em dessecador, foram pesadas para a determinação da porcentagem de cinza insolúvel em ácido.

Com os resultados laboratoriais, foram determinados os valores de energia metabolizável aparente (EMA) da dieta Referência e da Dieta Teste, e como base nestas foi calculada a EMA do milho de acordo como Sakomura \& Rostagno (2007). Também foram determinadas a taxa de recuperação do indicador nas excretas e os desvios entre as replicatas de análises de CIA.

Os resíduos foram submetidos à avaliação de homogeneidade e normalidade, removidos os outliers identificados e em seguida realizou-se à análise de variância (ANOVA) pelo procedimento GLM do SAS. Quando o fator foi significativo na ANOVA, foi utilizado o teste de Duncan ao nível de $5 \%$ de probabilidade.

OS cálculos foram feitos de acordo com as seguintes fórmulas: 
Cálculo da EMA do milho pelo procedimento da coleta total:

$$
\begin{aligned}
& \mathrm{EMA}_{\mathrm{RT}}(\mathrm{kcal} / \mathrm{g} \mathrm{MS})=\frac{\text { EB Ing. }(\mathrm{kcal}) \text { - EB Exc. (kcal) }}{\mathrm{MS} \text { Ing. }(\mathrm{g})} \\
& \text { EB Ing. (kcal) - EB Exc. (kcal) } \\
& \mathrm{EMA}_{\mathrm{RR}}(\mathrm{kcal} / \mathrm{g} \mathrm{MS})=\frac{\mathrm{MS} \text { Ing. }(\mathrm{g})}{\mathrm{-an}} \\
& \text { EMA milho }(\mathrm{kcal} / \mathrm{g} \mathrm{MS})=\mathrm{EMA}_{\mathrm{RR}}+\left(\begin{array}{c}
\mathrm{EMA}_{\mathrm{RT}}-\mathrm{EMA}_{\mathrm{RR}} \\
\text { \% } \\
\text { \% subst }
\end{array}\right.
\end{aligned}
$$

$\%$ subst $=\%$ inclusão $*$ \%MS milho / \%MS ração

Cálculo da EMA do milho pelo procedimento da coleta parcial:

EMA $(\mathrm{kcal} / \mathrm{Kg} \mathrm{MN})=\mathrm{EB} / \mathrm{kg}$ MS ração (kcal) - $(\mathrm{EB} / \mathrm{kg} \mathrm{MN}$ excretada (kcal) x FI) Em que:

$$
\mathrm{FI}=\frac{[\mathrm{CAI}] \text { na dieta }}{-[\mathrm{CAI}] \text { na excreta }}
$$

Cálculo da taxa de recuperação do indicador nas excretas Em que:

$$
\text { Taxa de Recuperação }=\frac{\text { Quantidade de indicador excretado }(\mathrm{g})}{\text { Quantidade de indicador ingerido }(\mathrm{g})}
$$

\section{RESULTADOS E DISCUSSÃO}

Não houve diferença significativa $(\mathrm{P}>0,05)$ entre a energia do milho pelos procedimentos de coleta (Tabela 2), com as técnicas utilizadas, indicando que a cinza insolúvel em ácido pode ser utilizada como indicador para predizer a EMA deste ingrediente. Estes resultados corroboram com os encontrados por Barbosa et al. (2008) e Santos et al. (2008) que, demonstraram que cinza insolúvel em ácido (CIA) apresenta boas estimativas de digestibilidade dos nutrientes e da energia metabolizável de dietas. Entretanto Dourado et al. (2010) verificaram que o uso de CIA superestimou a EMA do milho, porém foi adequada para determinação da EMA da dieta referência.

Observou-se que os métodos de análise apresentaram grande variabilidade, o que pode ser evidenciado por meio de observação do desvio padrão da média da EMA do milho (na matéria natural) estimada pelas diferentes técnicas analíticas de CIA, que oscilaram entre 88 a $229 \mathrm{kcal} / \mathrm{kg}$. Biologicamente essa variação pode causar uma grande diferença no desempenho dos animais, pois a estimativa da energia metabolizável do milho por meio de coleta parcial utilizando os métodos analíticos I, II e IV subestimou a 
energia do milho em 3,52\%; 1,73\% e $2,65 \%$, ou seja, uma diferença inferior de $110 ; 54$ e $83 \mathrm{kcal} / \mathrm{kg}$, respectivamente, em relação à coleta total de excretas.

Por outro lado, quando se utilizou o método III, a EMA do milho foi relativamente superestimada, em $112 \mathrm{kcal}$. Essas observações evidenciam que a correta estimativa do indicador está diretamente correlacionada com a técnica analítica empregada para determinação da concentração da CIA nas dietas e excretas, entretanto a EMA do milho pelos diferentes métodos de análise, não apresentaram diferenças estatísticas.

Tabela 2. Probabilidades obtidas na análise de variância e valores médios de Energia Metabolizável na Matéria Seca (EMAMS) e na Matéria Natural (EMAMN) do milho determinados pelo procedimento de coleta total e de coleta parcial (utilizando cinza insolúvel em ácido analisada por diferentes métodos), com respectivos desvios padrões

\begin{tabular}{lcc}
\hline \multirow{2}{*}{ Tratamento } & EMAMN & EMAMS \\
\cline { 2 - 3 } & \multicolumn{2}{c}{ Kcal/Kg de milho } \\
\hline Coleta Total & $3121 \pm 125$ & $3316 \pm 133$ \\
Coleta Parcial (Método I) & $3011 \pm 210$ & $3200 \pm 223$ \\
Coleta Parcial (Método II) & $3067 \pm 229$ & $3259 \pm 243$ \\
Coleta Parcial (Método III) & $3233 \pm 88$ & $3435 \pm 94$ \\
Coleta Parcial (Método IV) & $3038 \pm 127$ & $3229 \pm 135$ \\
\hline Probabilidade & $0,1838^{\text {ns }}$ & $0,1834^{\text {ns }}$ \\
\hline CV (\%) & 5,32 & 5,33
\end{tabular}

Houve diferença significativa $(\mathrm{P}<0,05)$ entre os procedimentos de coleta para a energia da dieta referência (Tabela 3), com as técnicas utilizadas, indicando que a cinza insolúvel em ácido pode ser utilizada como indicador para predizer a EMA da dieta, por meio de utilização dos métodos I, II e IV, pois os mesmos não apresentaram diferença estatística, entre o procedimento padrão de coleta total de excretas.

O método III superestimou a EMA da dieta em 3,16\%. Apesar da dieta referência ter sido utilizada como meio (metodologia) para a determinação da EMA do milho, a análise estatística da mesma foi realizada, pois a composição da dieta referência está de acordo com a exigência do animal. Dourado et al. (2010) relatam que a taxa de recuperação de indicadores da dieta referencia é próxima a $100 \%$, indicando que a estimativa de EMA com o uso de CIA é melhor quando as dietas estão balanceadas, quando comparadas com dietas teste.

Houve diferença significativa $(\mathrm{P}<0,05)$ entre os métodos de análise da CIA para a taxa de recuperação do indicador nas dietas (Tabela 4), indicando que a cinza insolúvel em acido pode ser utilizada como indicador, pois apresentou taxas de recuperação próximas à $100 \%$. Segundo Zeoula et al. (2002) a CIA é um indicador eficiente uma vez que os autores encontraram valores de recuperação fecal igual a $100 \%$, quando comparados com a coleta total de excreta. 
Tabela 3. Probabilidades obtidas na análise de variância e valores médios de Energia Metabolizável na Matéria Seca (EMAMS) e na Matéria Natural (EMAMN) da dieta referência (DR) determinados procedimento de coleta total e de coleta parcial (utilizando cinza insolúvel em ácido analisada por diferentes métodos), com respectivos desvios padrões

\begin{tabular}{lcc}
\hline \multirow{2}{*}{ Tratamento } & EMAMN & EMAMS \\
\cline { 2 - 3 } & \multicolumn{2}{c}{ Kcal/Kg de ração $^{1}$} \\
\hline Coleta Total & $2907 \pm 36^{\mathrm{bc}}$ & $3132 \pm 40^{\mathrm{c}}$ \\
Coleta Parcial (Método I) & $2850 \pm 41^{\mathrm{c}}$ & $3100 \pm 49^{\mathrm{c}}$ \\
Coleta Parcial (Método II) & $2908 \pm 64^{\mathrm{bc}}$ & $3162 \pm 70^{\mathrm{bc}}$ \\
Coleta Parcial (Método III) & $2999 \pm 45^{\mathrm{a}}$ & $3259 \pm 45^{\mathrm{a}}$ \\
Coleta Parcial (Método IV) & $2956 \pm 50^{\mathrm{ab}}$ & $3214 \pm 53^{\mathrm{ab}}$ \\
\hline Probabilidade & $0,0002^{*}$ & $0,0001^{*}$ \\
\hline CV (\%) & 1,65 & 1,66 \\
${ }^{\mathrm{T}}$ Médias na coluna seguidas de letras diferentes, diferem entre si $(\mathrm{P}<0,05)$ pelo teste de Duncan. ${ }^{*} \mathrm{P}<0,05$.
\end{tabular}

Tabela 4. Probabilidades obtidas na análise de variância e valores médios para a taxa de recuperação da cinza insolúvel em ácido (CIA), nas excretas das aves alimentadas com as duas dietas utilizadas

\begin{tabular}{lcc}
\hline Tratamento & Dieta Referência & Dieta Teste \\
\cline { 2 - 3 } Análise da CIA & Taxa de recuperação do indicador $(\%)^{1}$ \\
\hline Método I & $97,47 \pm 6,80^{\mathrm{c}}$ & $93,87 \pm 8,96^{\mathrm{c}}$ \\
Método II & $102,84 \pm 4,76^{\mathrm{bc}}$ & $99,77 \pm 6,84^{\mathrm{bc}}$ \\
Método III & $112,41 \pm 4,24^{\mathrm{a}}$ & $113,12 \pm 2,78^{\mathrm{a}}$ \\
Método IV & $107,71 \pm 4,91^{\mathrm{ab}}$ & $101,37 \pm 2,26^{\mathrm{b}}$ \\
\hline Probabilidade & $0,0006^{*}$ & $0,0002^{*}$ \\
\hline CV (\%) & 5,01 & 5,79 \\
${ }^{\mathrm{T}}$ Médias na coluna seguidas de letras diferentes, diferem entre si $(\mathrm{P}<0,05)$ pelo teste de Duncan. ${ }^{*} \mathrm{P}<0,05$.
\end{tabular}

Um bom indicador é caracterizado por ser uma substância conhecida, não tóxica, inalterada durante a passagem pelo intestino, que não exerça influência sobre os processos fisiológicos no trato digestório, não se associe a outros nutrientes, devendo ser totalmente recuperado nas excretas (KOBT \&LUCKEY, 1972). No entanto vários são os problemas associados com o uso de indicadores, não havendo um indicador que atenda de forma completa todos os critérios, principalmente ao que se refere à recuperação das excretas, assim devem-se estabelecer determinados critérios que forneçam subsídios para escolha de um indicador mais adequado com fácil aplicação e recuperação adequada de acordo com a dieta utilizada, custo e facilidade de analise (BERCHIELLI et al., 2005).

De acordo com os resultados, o método que apresentou maior taxa de recuperação foi o método III, indicando que este método pode superestimar a EMA da dieta ou do ingrediente. No entanto, os métodos I, II e IV não apresentaram diferenças estatísticas entre si, além de apresentar taxas mais próximas do recomendável, que seria em torno de $100 \%$.

Alguns fatores podem afetar a taxa de recuperação, dentre eles a quantidade de indicador incluída nas dietas. 
Segundo Lobo et al. (2001) as médias ( \pm desvio padrão) dos índices de recuperação para CIA foi de 93,9\% $( \pm 17,7)$. Para Bergero et al. (2004) encontram resultados positivos para o uso deste indicador, da mesma forma Kavanagh et al. (2001) o uso da CIA é um marcador rápido e confiável para mensuração de digestibilidade e oferece uma alternativa para o método de coleta total. No entanto valores contraditórios foram encontrados (PALANDER et al., 2010) para estimativa de fluxo de matéria seca fecal sub ou superestimando os coeficientes de digestibilidade. Segundo Sales \& Janssens (2003) o uso da CIA pode acarretar possíveis superestimações do indicador e diferenças na taxa de recuperação destes nas excretas. Desta forma pode-se relacionar que a composição dos ingredientes pode interferir nas estimativas com uso de indicadores, visto que a quantidade de alguns componentes, como a fibra, pode afetar a taxa de passagem e consequentemente a taxa de recuperação do indicador.

Houve diferença significativa $(\mathrm{P}<0,05)$ entre os métodos de análise da CIA do desvio-padrão das análises em duplicata e/ou triplicata de cinza insolúvel em ácido (CIA) nas excretas das aves alimentadas com a dieta teste (Tabela $5)$, indicando que existe uma grande variação da precisão das análises de cinza insolúvel em ácido pelas quatro técnicas utilizadas. De acordo com Brito et al. (2003) a precisão pode ser estimada pelas medidas de dispersão, como desvio-padrão, variância e coeficiente de variação e a exatidão pelo cálculo do erro absoluto e relativo, entre outras. A técnica que teve melhor precisão baseando-se no desvio padrão foi o método analítico III e IV.

Tabela 5. Probabilidades obtidas na análise de variância e valores médios do desviopadrão das análises de cinza insolúvel em ácido (CIA), nas excretas das aves alimentadas com as duas dietas utilizadas

\begin{tabular}{lcc}
\hline Tratamento & Dieta Referência & Dieta Teste \\
\cline { 2 - 3 } Análise da CIA & Desvio-padrão das replicatas das análises \\
\hline Método I & 0,123 & $0,187 \mathrm{~b}$ \\
Método II & 0,190 & $0,306 \mathrm{a}$ \\
Método III & 0,128 & $0,059 \mathrm{c}$ \\
Método IV & 0,110 & $0,092 \mathrm{bc}$ \\
\hline Probabilidade & $0,6471^{\mathrm{ns}}$ & $0,001^{*}$ \\
CV (\%) & 85,01 & 65,35 \\
\hline
\end{tabular}

$\mathrm{N} \mathrm{s}=$ não significativo pelo teste de Duncan a $5 \%$ de probabilidade.

${ }^{1}$ Médias na coluna seguidas de letras diferentes, diferem entre si $(\mathrm{P}<0,05)$ pelo teste de Duncan. ${ }^{*} \mathrm{P}<0,05$

Além dos resultados analíticos é importante ressaltar as condições dos equipamentos, pois alguns meses após a execução das análises verificou-se deterioração da centrifuga e do banhomaria utilizados (nas análises dos métodos I, II e III), provavelmente decorrente do vapor ácido liberado. Desta forma, o método IV seria o mais recomendado em função do conjunto de características favoráveis e facilidade de realização.

Assim pode-se inferir que a estimativa da energia metabolizável (EMA) do milho pela técnica de coleta parcial, com uso de cinza insolúvel em ácido (CIA) analisada por diferentes métodos, foi semelhante à de coleta total, 
demonstrando que a CIA pode ser utilizadas para estimar a EMA do milho. Os métodos de análise de CIA III e IV apresentaram melhor precisão analítica que os demais. Os métodos de análise de CIA II e IV apresentaram melhores taxas de recuperação próximas ao ideal.

Os métodos de análise da cinza insolúvel em ácido testados podem ser usados como indicador na determinação da energia metabolizável do milho para aves.

\section{REFERENCIAS}

BARBOSA, N.A.A.; SAKOMURA, N.K.; FERNANDES, J.B.K.;

DOURADO, L.R. B. Enzimas exógenas no desempenho e na digestibilidade ileal de nutrientes em frangos de corte.

Pesquisa Agropecuária Brasileira, v.43, p.755-762, 2008.

BERCHIELLI, T.T.; ANDRADE, P.; FURLAN, C.L. Comparação de marcadores para estimativas de produção fecal e de fluxo de digesta em bovinos. Revista Brasileira de Zootecnia, v.34, p.987-996, 2005.

BERGERO, D.; MIRAGLIA, N.; ABBA, C.; POLIDORI, M. Apparentof digestibility mediterranean forages determined by total collection of faeces and acid-insoluble ash as internal marker. Livestock Production Science, v.85, p.235-238, 2004.

BRITO, N.M.; AMARANTE, O.P.; POLESE, L.; RIBEIRO, M.L. Avaliação da exatidão e da precisão de métodos de análise de resíduos de pesticidas mediante ensaios de recuperação. Pesticidas: Revista de Ecotoxicologia e Meio Ambiente, v.12, p.155-168, 2003.
D’AGOSTINI, P.; GOMES, P.C.; ALBINO, L.F.T.; ROSTAGNO, H.S.; SA, S.M. Valores de composição química e energética de alguns alimentos para Aves. Revista Brasileira de Zootecnia, v.33, p.128134, 2004.

DOURADO, L.R.B.; SIQUEIRA, J.C.; SAKOMURA, N.K.; PINHEIRO, S.R.F.; MARCATO, S.M.; FERNANDES, J.B.K.; SILVA, J.H.V. Poultry feed metabolizable energy determination using total or partial excreta collection methods. Brazilian Journal of Poultry Science, v.12, p.129-132, 2010.

FREITAS, E.R.; SAKOMURA, N.K.; EZEQUIEL, J.M.B.; NEME, R.; MENDONÇA, M.O. Energia metabolizável de alimentos na formulação de ração para frangos de corte. Pesquisa Agropecuaria Brasileira, v.41, p.107-115, 2006.

KAVANAGH, S.; LYNCH, P.B; MARA, O.F.; CAFREY, P.J. A comparison of total collection and marker technique for the measurement of apparent digestibility of diets for growing pigs. Animal Feed Science and Technology, v.89, p.49-58, 2001.

KOBT, A.R.; LUCKEY, T.D. Markers in nutrition. Nutrition Abstracts and Reviews, v.42, p.813-845, 1972.

LOBO, M.S.; RESENDE, C.S.A.; SALIBA, S.O.E.; SAMPAIO, I.M.B. Coeficientes de digestibilidade aparente pelos métodos de indicadores e coleta total de fezes em cães. Arquivo Brasileiro de Medicina Veterinária e Zootecnia, v.53, p.691-694, 2001.

PALANDER, S.; NASI, M.; PALANDER, P. Digestibility and energy volue of cereal-based diets in relation to 
digesta viscosity and retention time in turkey and chickens at different ages estimated with different markers.

Archives of Animal Nutrition, v. 64, p.238-253, 2010.

ROSTAGNO, H.S.; ALBINO, L.F.T.; DONZELE, J.L.; GOMES, P.C.; OLIVEIRA, R.F.; LOPES, D.C.; FERREIRA, A.S.; BARRETO, S.L.T. Composição de alimentos e exigências nutricionais: tabelas brasileiras para aves e suínos. Viçosa, MG: UFV, 2005, p.186.

SAKOMURA, N.K.; ROSTAGNO, H.S. Métodos de pesquisa em nutrição de monogástricos. Jaboticabal: FUNEP, 2007. 283p.

SALES, J.; JANSSENS, G.P.J. The use of markers to determine energy matabolizability and nutrient digestibility in avian species. World's Poultry Science Journal, v.59, p.214-237, 2003.

SANTOS, F.R.; HRUBY, M.; PIERSON, E.E.M.; REMUS, J.C.; SAKOMURA,N.K. Effect of phytase supplementation in diets on nutrient digestibility and performance in Broiler Chicks. Journal Applied Poultry Research, v.17, p.191-201, 2008.
SILVA, D.J.; QUEIROZ. A. Análise de alimentos: métodos químicos $\mathrm{e}$ biológicos. 3.ed. Viçosa: UFV, 2002. $235 \mathrm{p}$.

TORRES, L.C.L.; FERREIRA, M.A.; GUIM, A.; VILELA, M.S.; GUIMARÃES, A.G.; SILVA, E.C. Substituição da palma-gigante por palma-miúda em dietas para bovinos em crescimento e avaliação de indicadores internos. Revista Brasileira de Zootecnia, v.38, p.2264-2269, 2009.

Van KEULEN, J.; YOUNG, B.A. Evaluation of acid-insoluble ash as natural marker in ruminant digestibility studies. Journal of Animal Science, v.4, p.282-287, 1977.

ZEOULA, L.M.; PRADO, I.N.; DIAN, P.H.M.; GERON, J.J.V.; CALDAS, F.S.; MARQUES, J.A.; FALCAO, A.J.S. Recuperação fecal de indicadores internos avaliados em ruminantes.

Revista Brasileira de Zootecnia, v.31, p.1865-1874, 2002.

Data de recebimento: $11 / 07 / 2012$

Data de aprovação: 14/01/2013 